Ebisu Ebisu

Études japonaises Études japonaises

$57 \mid 2020$

Les architectes de l'ère Heisei (1989-2019). Rôles, statuts, pratiques et productions

\title{
CORRAL Jeremy, Japanoise. Extrémismes \& Entropie
}

Dijon, Les Presses du réel, Collection Délashiné, 2019, 359 p.

\section{Véronique Brindeau}

\section{(2) OpenEdition}

\section{Journals}

Édition électronique

URL : http://journals.openedition.org/ebisu/5462

DOI : $10.4000 /$ ebisu.5462

ISSN : 2189-1893

Éditeur

Institut français de recherche sur le Japon à la Maison franco-japonaise (UMIFRE 19 MEAE-CNRS)

Édition imprimée

Date de publication : 15 décembre 2020

Pagination : 460-462

ISSN : 1340-3656

\section{Référence électronique}

Véronique Brindeau, « Corral Jeremy, Japanoise. Extrémismes \& Entropie », Ebisu [En ligne], 57 | 2020, mis en ligne le 20 décembre 2020, consulté le 29 mars 2021. URL : http://journals.openedition.org/ ebisu/5462 ; DOI : https://doi.org/10.4000/ebisu.5462 


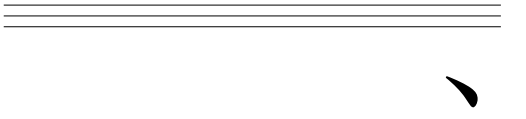

() Corral Jeremy, Japanoise. Extrémismes \& Entropie, Dijon, Les Presses du réel, Collection Délashiné, 2019, 359 p.

Au-delà d'une somme extrêmement documentée sur un mouvement artistique largement méconnu de la critique en langue française, et dont l'accès a longtemps été restreint à un public d'amateurs exclusifs du genre, l'ouvrage de Jeremy Corral, écrit dans une langue ferme et précise, ouvre des perspectives rigoureuses sur la japanoise en reconstituant son histoire, ses pratiques, ainsi que son rapport d'articulation avec les arts contemporains.

Anticonformiste à l'extrême, marquée par un goût exacerbé pour l'excès, la provocation et une organicité fondamentale, la musique noise constitue l'un des phénomènes majeurs de l'histoire des avant-gardes et de la contre-culture au Japon depuis la fin des années 1970. Au sein de ce courant foisonnant, Jeremy Corral s'attache à préciser ici les contours de la notion de japanoise ainsi que les éventuelles spécificités induites par une telle dénomination, en retraçant sa genèse et en soulignant les zones d'influences et de divergences avec diverses musiques "alternatives " - punk, musique industrielle, rock électronique progressif, jazz fusion entre autres. L'auteur, qui achève une thèse sur les débuts de la musique électroacoustique au Japon, se réfère à un vaste corpus documentaire constitué à la fois d'ouvrages critiques, d'entretiens publiés sur des sites Internet très spécialisés et d'une multiplicité de traces liées à ce genre hors normes : comptes rendus de prestations scéniques, photographies, affiches, supports divers d'enregistrements qui donnent lieu dans cet ouvrage à une analyse d'ordre sémiologique.

Après un préambule situant l'évolution globale de la notion de bruit en musique et son ascension avec l'avènement des avant-gardes au $\mathrm{Xx}^{\mathrm{e}}$ siècle, Jeremy Corral présente un ensemble significatif de groupes et de personnalités de la scène artistique japonaise engagés dans le courant directement issu des musiques dites "bruitistes " qui se développe au Japon à partir de la fin des années 1970 . Tout en se réappropriant le " matériau d'investigation des artistes d'avant-garde que sont Luigi Russolo, Pierre Schaeffer ou encore John Cage» (p. 24), la noise rejette les formes, codes musicaux et cadres constitués jusque-là en vigueur.

S'appuyant en particulier sur les travaux de Paul Hegarty dans Noisel music - A History, l'auteur poursuit 
sa présentation en parcourant les différentes modalités du rapport de la musique au bruit, tout spécialement via l'adjonction intentionnelle de sonorités parasites, dont témoigne maints aspects de la musique dite traditionnelle au Japon (tels le souffle dans le jeu de la flûte shakuhachi 尺八 ou le son "zingué» de certains luths biwa 琵琶). La section intitulée « Bruits. Graphies - Fabriques de l'entropie " brosse un portrait détaillé de dix groupes marquants de la scène noise au Japon. Ce précieux état des lieux - il n'existe à ce jour aucun ouvrage en français permettant d'accéder à une telle collecte d'informations - est établi selon une approche que l'auteur définit lui-même comme "kaléidoscopique ". Sont ici rassemblés des entretiens avec des artistes, transcrits et traduits par l'auteur, ainsi qu'un abondant corpus iconographique documentant des prestations scéniques et révélant la variété des supports de musique enregistrée, parfois incongrus - sans omettre un démontage minutieux des différents mécanismes de distribution et de réception de la noise. Chaque présentation est suivie d'une discographie sélective particulièrement fournie.

La spécificité présumée de l'aire culturelle du Kansai est abordée dans «Kansai no wave/Kansai no tale, d'un récit à l'histoire». Le regard de Jeremy Corral maintient ici une distance critique vis-à-vis de l'objet de son étude qui lui permet de prendre en compte des stratégies de distribution éventuellement encouragées par les artistes eux-mêmes: ainsi de l'élaboration d'une "narration" marquant une spécificité du Kansai, revendiquée par l'une des entités les plus emblématiques de la noise, Hijōkaidan 非常階段 (Escalier de secours), "à l'origine du récit le plus communément répandu de la naissance et du développement de la noise » (p. 149), qui fait ici l'objet d'une analyse spécifique.

Décomposée en ses deux éléments constitutifs, l'expression forgée japanoise suppose un écart entre une identité japonaise présumée (japa-) et un courant mondialisé (-noise), écart que Jeremy Corral analyse finement comme pouvant résulter aussi bien d'une réception internationale que d'une recherche délibérée de particularisme par les acteurs de ce mouvement. Après une première génération de musiciens noise qui a posé les fondements d'un travail sur le bruit, d'autres artistes ont en effet réinterprété cette approche en approfondissant la coupure avec la création européenne et américaine et en produisant une surabondance de signes, pour défaire l'ordre des agencements catégoriels. Il apparaît en réalité qu'une multitude de pratiques noise coexistent au Japon, fondées 
sur une quête nécessairement inatteignable d'entropie, autrement dit de désorganisation: le principe même d'une « œuvre" noise, fût-elle établie sur le principe global d'une saturation de l'intensité sonore, voire sur l'instauration d'un véritable "mur de son " coupé net en bout de piste, d'un refus de toute convention et de toute narration, ne peut que constituer une contradiction dans les termes - sauf à détruire les traces de cette expression dans l'instant même de son écoute ou de sa performance (ce qu'opère, à la lettre, l'artiste Yamano.uchi Juntarō 山之内純太郎, en incinérant les exemplaires du single Aijin 愛人 au moment de sa sortie).

Objet du dernier chapitre de l'ouvrage ("De l'entropie comme [dés] ordonnancement du monde ") le projet Merzbow d'Akita Masami 秋田昌美, visant 《 une nouvelle totalité non catégorielle", s'inscrit dans son intitulé même dans la continuité de l'œuvre Merzbau de l'artiste allemand Kurt Schwitters (1887-1948), issu du dadaïsme, qui se réapproprie les rebuts de la société urbaine et industrielle. Le projet d'Akita apparaît non seulement comme l'une des approches les plus prolifiques au sein de la noise et l'une des plus étudiées de la création japonaise, mais aussi comme l'une des plus exigeantes, et peut être envisagé dans toutes ses dimensions comme "un travail sans cesse remis à jour de l'impermanence".

En conclusion de son analyse de la japanoise, Jeremy Corral examine le déploiement de cette modalité spécifique, au Japon, de modeler le bruit de la noise, non pas en l'essentialisant comme une pratique culturelle locale singulière et isolée, mais en la considérant dans sa relation avec certaines des avant-gardes artistiques majeures du $\mathrm{Xx}^{\mathrm{e}}$ siècle : la pensée du musicien John Cage, d'une part, envisageant comme musique tout phénomène sonore porté à l'attention par une exposition particulière; le groupe Fluxus d'autre part, brouillant toute démarcation entre les sphères de l'art et du quotidien. Deux courants dont on sait combien ils reçurent un accueil éminemment favorable dans le monde de la musique et de l'art contemporain au Japon.

Véronique BrindeAu Chargée de cours à l'Inalco 